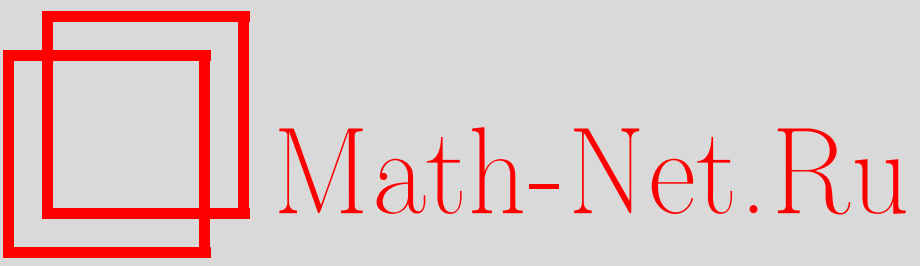

В. Б. Васильев, О некоторых операторных семействах, Итоги науки и техн. Сер. Соврем. мат. и ее прил. Темат. обз., 2019, том 168, 26-32

DOI: https://doi.org/10.36535/0233-6723-2019-168-26-32

Использование Общероссийского математического портала Math-Net.Ru подразумевает, что вы прочитали и согласны с пользовательским соглашением

http://www.mathnet.ru/rus/agreement

Параметры загрузки:

IP: 52.23 .180 .231

26 апреля 2023 г., 08:34:12 


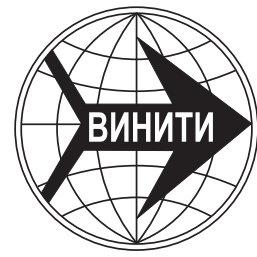

ИТОГИ НАУКИ И ТЕХНИКИ.

Современная математика и ее приложения.

Тематические обзоры.

Том 168 (2019). C. 26-32

DOI: $10.36535 / 0233-6723-2019-168-26-32$

УДК 517.983

\title{
О НЕКОТОРЫХ ОПЕРАТОРНЫХ СЕМЕЙСТВАХ
}

\author{
(C) 2019 г. $\quad$ В. Б. ВАСИЛЬЕВ
}

\begin{abstract}
АннотАция. Предлагается абстрактная схема исследования специальных операторов, которая применяется далее для изучения эллиптических псевдодифференциальных операторов и связанных с ними краевых задач на многообразиях с негладким краем. В частности, граница может содержать конические точки, ребра различных размерностей, и в некоторых случаях точки пика. С помощью таких конструкций описываются корректные постановки краевых задач для эллиптических псевдодифференциальных уравнений на указанных многообразиях в пространствах Соболева-Слободецкого.
\end{abstract}

Ключевые слова: оператор локального типа, операторный символ, эллиптичность, фредгольмовость, индекс, псевдодифференциальный оператор.

\section{ON CERTAIN OPERATOR FAMILIES}

\author{
(c) 2019 V. B. VASILYEV
}

\begin{abstract}
In this paper, we propose an abstract scheme for the study of special operators and apply this scheme to examining elliptic pseudodifferential operators and related boundary-value problems on manifolds with nonsmooth boundaries. In particular, we consider cases where boundaries may contain conical points, edges of various dimensions, and even peak points. Using the constructions proposed, we present well-posed formulations of boundary-value problems for elliptic pseudo-differential equations on manifolds discussed in Sobolev-Slobodecky spaces.
\end{abstract}

Keywords and phrases: local-type operator, operator symbol, ellipticity, Fredholm property, index, pseudodifferential operator.

AMS Subject Classification: 35S05, 47B37

1. Введение. В теории псевдодифференциальных операторов и связанных с ними уравнений и краевых задач ключевую роль играет понятие символа псевдодифференциального оператора. В терминах символа определяется сам псевдодифференциальный оператор, условия эллиптичности и фредгольмовости. Принято считать символ некоей функцией, определенной на какой-либо геометрической структуре (в случае псевдодифференциальных операторов на гладких многообразиях - это кокасательное расслоение). Все арифметические операции, производимые в алгебре операторов (факторизованной по идеалу компактных операторов), наследуются алгеброй символов, после чего все внимание уделяется исследованию алгебры символов. Последняя может оказаться коммутативной (скалярный случай), а может быть и некоммутативной (матричный случай). Подход, связанный с операторными алгебрами, наверное, наиболее популярен в последние два-три десятилетия (см. $[5,10,13,14])$, однако имеются и аналитические рассмотрения (см. $[4,6,9,11,12])$. Основная проблема, которой посвящены эти работы-описание условий фредгольмовости рассматриваемых операторов и вычисление их индекса.

Работа выполнена при поддержке Министерством образования и науки Российской Федерации (проект № $1.7311 .2017 / 8.9)$. 
В настоящей работе предлагается операторный подход к этой проблеме, основанный на рассмотрениях (см. [7]) для специальных операторов локального типа (см. ниже), к которому можно отнести широкие классы псевдодифференциальных операторов. Некоторые предварительные наработки, связанные с этим исследованием, содержатся в работах автора (см. $[1-3,15])$.

2. Операторы локального типа и огибающие. Приведем некоторые общие положения, связанные со специальными классами операторов, основанные на понятиях и конструкциях И. Б. Симоненко (см. [7]).

2.1. Основные понятия. Пусть $H_{1}, H_{2}$ - гильбертовы (банаховы) пространства функций, определенных на компактном многообразии (возможно, с краем) $M, A: H_{1} \rightarrow H_{2}$-линейный ограниченный оператор. Следуя [7] введем следующее определение.

Определение 1. Оператор $A$ называется оператором локального типа, если оператор $P_{U} A P_{V}$ является компактным для любых двух компактных непересекающихся множеств $K_{1}, K_{2} \subset M$, $K_{1} \cap K_{2}=\emptyset ; P_{K}$ обозначает проектор на множество $K$; точнее, для всех $f(x) \in H_{1}\left(H_{2}\right)$

$$
\left(P_{K} f\right)(x)= \begin{cases}f(x), & x \in K \\ 0, & x \in M \backslash K .\end{cases}
$$

Всюду ниже будем рассматривать только операторы локального типа.

Обозначим символом $\||A \|| \mid$ существенную норму оператора $A$,

$$
\|\mid A\| \equiv \inf \|A+T\|,
$$

где точная нижняя грань берется по всем компактным операторам $T: H_{1} \rightarrow H_{2}$.

Определение 2. Оператор $A_{x}: H_{1} \rightarrow H_{2}$ называется локальным представителем оператора $A$ в точке $x \in M$, если для любого $\varepsilon>0$ найдется такая окрестность $U$ точки $x$ на многообразии $M, x \in U \subset M$, что выполняется неравенство

$$
\left\|P_{U}\left(A-A_{x}\right) \mid\right\|<\varepsilon .
$$

Обозначение $A \stackrel{x}{\sim} A_{x}$.

Определение 3. Символом оператора $A$ называется оператор-функция $A(x): M \rightarrow$ $\left\{A_{x}\right\}_{x \in M}$, определяемая его локальными представителями.

Нетрудно убедиться (см. [7]), что такое определение символа сохраняет все свойства символического исчисления. Именно, с точностью до компактного слагаемого

- произведению и сумме двух операторов соответствует произведение и сумма их локальных представителей;

- сопряженному оператору соответствует сопряженный его локального представителя;

- фредгольмову оператору соответствует фредгольмов локальный представитель.

Под оператором Фредгольма (фредгольмовым оператором) мы будем понимать линейный ограниченный оператор с конечным индексом. В [7] приведен следующий критерий фредгольмовости: оператор А фредгольмов тогда и только тогда, когда символ $A(x)$ состоит из фредгольмовых операторов.

Пусть задано семейство операторов $\left\{A_{x}\right\}_{x \in M}$.

Определение 4. Семейство $\left\{A_{x}\right\}_{x \in M}$ называется локально непрерывным, если для любых $\varepsilon>$ 0 и $x_{0} \in M$ найдется такая окрестность $U \subset M$ точки $x_{0}$, что для для каждой точки $x \in U$ выполняется неравенство

$$
\left\|P_{U}\left(A_{x}-A_{x_{0}}\right) \mid\right\|<\varepsilon .
$$

Определение 5. Оператор $A$ называется огибающим оператором семейства $\left\{A_{x}\right\}_{x \in M}$, если

$$
A \stackrel{x}{\sim} A_{x} \quad \forall x \in M
$$

В [7] доказано также существование единственного (с точностью до компактного) огибающего оператора для любого локально непреръьного семейства $\left\{A_{x}\right\}_{x \in M}$. 
2.2. Пересажсивание. Пусть $H_{1}^{\prime}, H_{2}^{\prime}$ - гильбертовы пространства, состоящие из определенных на $\mathbb{R}^{m}$ функций, $\widetilde{A}: H_{1}^{\prime} \rightarrow H_{2}^{\prime}$-линейный ограниченный оператор. Поскольку $M-$ компактное многообразие, у каждой точки $x \in M$ имеется окрестность $U \ni x$ и диффеоморфизм $\omega: U \rightarrow$ $D_{x} \subset \mathbb{R}^{m}, \omega(x) \equiv y$. Обозначим через $S_{\omega}$ следующий (локальный) оператор, действующий из $H_{k}$ в $H_{k}^{\prime}, k=1,2$. Для каждой функции $u \in H_{k}$, обнуляющейся вне $U$, имеем

$$
\left(S_{\omega} u\right)(y)= \begin{cases}u\left(\omega^{-1}(y)\right), & y \in D_{x}, \\ 0, & y \notin D_{x} .\end{cases}
$$

Следующее определение соответствует понятию квазиэквивалентности (см. [7]).

Определение 6. Локальным представителем оператора $A: H_{1} \rightarrow H_{2}$ в точке $x \in M$ называется такой оператор $\widetilde{A}: H_{1}^{\prime} \rightarrow H_{2}^{\prime}$, что для любого $\varepsilon>0$ существует окрестность $U_{j}$ точки $x \in U_{j} \subset M$, для которой выполняется следующее неравенство:

$$
\left\|g_{j} A f_{j}-S_{\omega_{j}^{-1}} \hat{g}_{j} \tilde{A} \hat{f}_{j} S_{\omega_{j}}\right\| \mid<\varepsilon
$$

для любой пары гладких функций $f_{j}, g_{j}$ с носителями в $\bar{U}_{j}, \hat{f}_{j}, \hat{g}_{j}$-их запись в локальных координатах.

3. Эллиптичность и фредгольмовость. Отправляясь от вышеприведенных фактов и учитывая дальнейшие применения к псевдодифференциальным операторам, мы дадим следующее определение.

Определение 7. Оператор $A$ называется эллиптическим, если его символ состоит из обратимых операторов.

Мы рассмотрим здесь случай, когда семейство $\left\{A_{x}\right\}_{x \in M}$ не является локально непрерывным в целом, однако сохраняет это свойство на отдельных (особых) подмногообразиях $M_{k} \subseteq M$ размерности $k=0,1, \ldots, m$. Так, подмногообразие $M_{0}$ представляет собой конечное объединение различных точек границы $\partial M$ многообразия $M, M_{n} \equiv M$ - это исходное многообразие, $M_{n-1} \equiv \partial M$, $M_{k} \subset M_{n-1}, k=0,1, \ldots, n-2$. Пусть на $M$ задано семейство $\left\{A_{x}\right\}_{x \in M}$. Мы будем предполагать, что это семейство локально непрерывно на $M \backslash \bigcup_{k=1}^{n-1} M_{k}$, причем существуют пределы $\lim _{x \rightarrow x_{k} \in M_{k}} A_{x}$, вообще говоря, не совпадающие с $A_{x_{k}}$, и локально непрерывно на каждом $M_{k} \backslash \bigcup_{j=0}^{k-1} M_{j}$ и существуют пределы $\lim _{x \rightarrow x_{j} \in M_{j}} A_{x}$, вообще говоря, не совпадающие с $A_{x_{j}}$.

Пусть теперь $A$-оператор, символом которого является описанное семейство $\left\{A_{x}\right\}_{x \in M}$.

Теорема 1. Эллиптический оператор всегда фредгольмов.

С помощью разбиения единицы на многообразии $M$ по эллиптическому символу $A(x)$ можно построить $n$ операторов $A_{j}$ согласно числу особых подмногообразий $M_{k}$, включая всю границу $\partial M$ и само многообразие $M$.

Теорема 2. Индекс фредгольмова оператора А представи́м формулой

$$
\text { Ind } A=\sum_{j=1}^{n} \operatorname{Ind} A_{j} \text {. }
$$

4. Псевдодифференциальные операторы на конических расслоениях. Опишем некоторые применения вышеописанной абстрактной схемы к исследованию псевдодифференциальных операторов и уравнений на многообразиях с негладким краем. Это удобнее сделать в контексте векторных расслоений (см. [6,8]), поскольку возникают трудности с определением кокасательного расслоения в точках негладкости края. 
4.1. Коническое расслоение. Под коническим расслоением $E$ над $M$ будем понимать расслоение в обычном смысле (см. $[6,8])$ с базой $M$ (многообразие), слоями $\mathbb{R}^{m}$ и локальной тривиализацией вида $U \times \mathbb{R}^{m}$, где окрестность $U$ диффеоморфна некоторому конусу в $m$-мерном пространстве. Эти конусы будут разными в зависимости от расположения точки базы. В частности, это будут конусы вида $\mathbb{R}^{m}, \mathbb{R}_{+}^{m}=\left\{x \in \mathbb{R}^{m}: x=\left(x^{\prime}, x_{m}\right), x_{m}>0\right\}, W^{k}=\mathbb{R}^{k} \times C^{m-k}\left(W^{0} \equiv C^{m}\right)$, где $C^{m-k}$ - острый выпуклый конус в $\mathbb{R}^{m-k}$, не содержащий целой прямой.

4.2. Псевдодифференииальный оператор на коническом расслоении. Здесь мы приведем конструкцию псевдодифференциального оператора $A$ на $m$-мерном компактном многообразии $M$ с краем, которая определяется заданной функцией $A(x, \xi),(x, \xi) \in E$.

Будем предполагать, что $A(x, \xi)$ непрерывно дифференцируема по $(x, \xi) \in E$ и удовлетворяет условию

$$
c_{1}(1+|\xi|)^{\alpha} \leqslant|a(x, \xi)| \leqslant c_{2}(1+|\xi|)^{\alpha},
$$

где $\alpha \in \mathbb{R}$ называется порядком оператора. Под $a(x, \xi),(x, \xi) \in U \times \mathbb{R}^{m}$, здесь понимается символ $A(x, \xi),(x, \xi) \in E$, записанный в локальных координатах. Порождающую функцию $A(x, \xi)$, определенную на коническом расслоении $E$, будем называть классическим символом оператора, и говорить, что классический символ эллиптический, если $A(x, \xi) \neq 0$ для всех $(x, \xi) \in E$.

На крае $\partial M$ многообразия $M$ выделены гладкие подмногообразия $M_{k}$ (особенности) размерности $0 \leqslant k \leqslant m-1$. Локальный представитель оператора $A$ в точке $x_{0} \in M$ на карте $U \ni x_{0}$ определяется формулой

$$
\left(A_{x_{0}} u\right)(x)=\iint_{D_{x_{0}}} \int_{\mathbb{R}^{m}} e^{i \xi \cdot(x-y)} a\left(x_{0}, \xi\right) u(y) d \xi d y, \quad x \in D_{x_{0}},
$$

и структура канонической области $D_{x_{0}}$ имеет различный вид в зависимости от расположения точки $x_{0}$ на многообразии $M$. Варианты канонических областей $D_{x_{0}}: \mathbb{R}^{m}, \mathbb{R}_{+}^{m}=\left\{x \in \mathbb{R}^{m}: x=\right.$ $\left.\left(x^{\prime}, x_{m}\right), x_{m}>0\right\}, W^{k}=\mathbb{R}^{k} \times C^{m-k}$, где $C^{m-k}$ - острый выпуклый конус в $\mathbb{R}^{m-k}$.

Определение 8. Семейство $\left\{A_{x}\right\}$ операторов (2) называется символом псевдодифференциального оператора $A$.

По заданному символу конструируется сам псевдодифференциальный оператор.

Если $M$-компактное многообразие, то на нем существует специальное разбиение единиць (см. $[1,6])$. Это означает, что для любого конечного открытого покрытия $\left\{U_{j}\right\}_{j=1}^{k}$ многообразия $M$ найдется такая система функций $\left\{\varphi_{j}(x)\right\}_{j=1}^{k}, \varphi_{j}(x) \in C^{\infty}(M)$, что

(i) $0 \leqslant \varphi_{j}(x) \leqslant 1$,

(ii) $\operatorname{supp} \varphi_{j} \subset U_{j}$,

(iii) $\sum_{j=1}^{k} \varphi_{j}(x)=1$.

Таким образом, имеем

$$
f(x)=\sum_{j=1}^{k} \varphi_{j}(x) f(x)
$$

для любой функции $f$, определенной на $M$.

На многообразии $M$ зафиксируем два конечных покрытия и два разбиения единицы, соответствующие этим покрытиям, $\left\{U_{j}, f_{j}\right\}_{j=1}^{n}$ и $\left\{V_{j}, g_{j}\right\}_{j=1}^{n}$, так что $\overline{U_{j}} \subset V_{j}$.

Определение 9. Псевдодифференциальным оператором $A$ на многообразии $M$ называется оператор, представимый в виде

$$
A=\sum_{j=1}^{n} S_{\omega_{j}^{-1}} \hat{f}_{j} \cdot \tilde{A}_{x_{j}} \cdot \hat{g}_{j} S_{\omega_{j}}+T
$$

где $T: H_{1} \rightarrow H_{2}$ - компактный оператор, $x_{j} \in U_{j}, \tilde{A}_{x_{j}}$ - символ из семейства $(2)$ в точке $x_{j}$. 


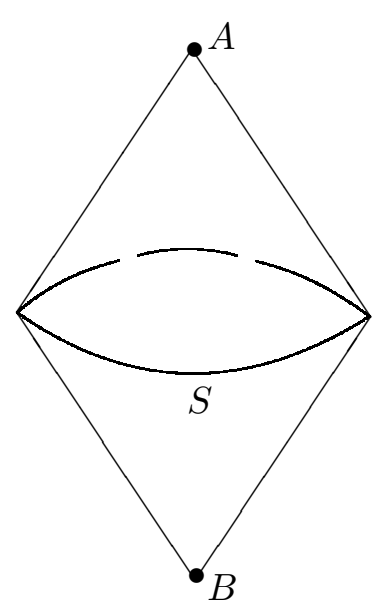

Рис. 1. Иллюстрация к теореме 2

Замечание 1. Определение 9 не зависит от выбора атласа, разбиения единицы и локальной системы координат в том смысле, что при такой замене к локальному представителю может лишь добавиться компактный оператор; это многократно встречалось ранее (см. $[6,7,9])$.

Оператор $A$ с символом, удовлетворяющим условию (1), удобно рассматривать в пространствах Соболева-Слободецкого $H^{s}(M)$ (см. [9]), где в качестве их локальных вариантов будут фигурировать пространства $H^{s}\left(D_{x_{0}}\right)$. Это пространство $H^{s}(M)$ строится с помощью разбиения единицы (см. [9]), и $A: H^{s}(M) \rightarrow H^{s-\alpha}(M)$ оказывается линейным ограниченным оператором локального типа.

Пример 1. В качестве иллюстрации к теореме 2 и определению 7 опишем структуру псевдодифференциального оператора на простом многообразии с негладкой границей, изображенном на рис. 1.

Оператор, определенный на таком многообразии локальными формулами (2), «склеивается» в пять различных операторов: одна огибающая $A_{1}$ по внутренним точкам «волчка», вторая огибающая $A_{2}$ - по точкам гладкости границы, третья огибающая $A_{3}$ - по кромке ребра, четвертая и пятая $A_{4}, A_{5}$ - отдельные операторы, соответствующие коническим точкам. Чтобы описать условия фредгольмовости, приходится предварительно выяснять условия фредгольмовости (в двух последних случаях - обратимости) всех пяти операторов.

5. Локальные индексы и фредгольмовость. Для того, чтобы сформулировать результат о фредгольмовости эллиптического (в смысле определения 7) псевдодифференциального оператора, необходимо ввести некоторые дополнительные характеристики классического символа эллиптического псевдодифференциального оператора (см. [1,2]).

Обозначим символом $\stackrel{*}{C}^{m-k}$ сопряженный конус для $C^{m-k}$ :

$$
\stackrel{*}{C}^{m-k}=\left\{x \in \mathbb{R}^{m}: x \cdot y>0 \forall y \in C^{m-k}\right\},
$$

$T\left( \pm \stackrel{*}{C^{m-k}}\right)$ обозначает радиальную трубчатую область над конусом $\pm \stackrel{*}{C^{m-k}}$, т.е. область многомерного комплексного пространства $\mathbb{C}^{m}$ вида $\mathbb{R}^{m} \pm \stackrel{*}{C^{m-k}}$.

Пусть функция $a(\xi), \xi \in \mathbb{R}^{m}$, удовлетворяет условию (1). Введем обозначения $\xi=\left(\xi^{\prime \prime}, \xi^{\prime}\right)$, $\xi^{\prime \prime}=\left(\xi_{1}, \ldots, \xi_{k}\right), \xi^{\prime}=\left(\xi_{k+1}, \ldots, \xi_{m}\right)$.

Определение 10. $k$-Волновой факторизацией функции $a(\xi)$ относительно конуса $C^{m-k}$ называется его представление в виде

$$
a(\xi)=a_{\neq}(\xi) a_{=}(\xi),
$$

где сомножители $a_{\neq}(\xi), a_{=}(\xi)$ удовлетворяют следующим условиям: 
1) $a_{\neq}(\xi), a_{=}(\xi)$ определены для всех значений $\xi \in \mathbb{R}^{m}$, кроме, возможно, точек вида $\mathbb{R}^{k} \times$ $\partial\left(\stackrel{*}{C}^{m-k} \cup\left(-\stackrel{*}{C}^{m-k}\right)\right)$

2) $a_{\neq}(\xi), a_{=}(\xi)$ допускают аналитическое продолжение в радиальные трубчатые области $T\left(\stackrel{*}{C}^{m-k}\right), T\left(-\stackrel{*}{C}^{m-k}\right)$ при почти всех $\xi^{\prime \prime} \in \mathbb{R}^{k}$ соответственно, для которых справедливы оценки

$$
\begin{gathered}
\left|a_{\neq}^{ \pm 1}\left(\xi^{\prime \prime}, \xi^{\prime}+i \tau\right)\right| \leqslant c_{1}(1+|\xi|+|\tau|)^{ \pm \varkappa_{k}}, \\
\left|a_{=}^{ \pm 1}\left(\xi^{\prime \prime}, \xi^{\prime}-i \tau\right)\right| \leqslant c_{2}(1+|\xi|+|\tau|)^{ \pm\left(\alpha-\varkappa_{k}\right)}
\end{gathered}
$$

для всех $\tau \in \stackrel{*}{C}^{m-k}$.

Число $\varkappa_{k} \in \mathbb{R}$ называется индексом $k$-волновой факторизации.

6. Эллиптичность, фредгольмовость и краевые задачи. Предположим, что символ оператора $A$ представляет собой локально непрерывное на $M_{k}, k=0,1, \ldots, m$, согласованное семейство операторов. В частности, это всегда справедливо, если функция $A(x, \xi)$, определенная на коническом расслоении, является непрерывно дифференцируемой вплоть до границы. Тогда (теорема об огибающей; см. [7]) по операторному символу (2) можно построить $n$ операторов $A_{k}$, и в случае фредгольмовости каждого из них, исходный оператор также будет фредгольмов $\mathrm{c}$ индексом, предписанным теоремой 2.

Обозначим через $\varkappa_{n-1}(x)$ индекс факторизации функции $A(x, \xi)$ в точке $x \in \partial M \backslash \bigcup_{k=0}^{m-2} M_{k}$ (см. [9]), $\varkappa_{k}(x)$ - индексы $k$-волновой факторизации относительно конуса $C_{x}^{m-k}$ в точках $x \in$ $M_{k}, k=0,1, \ldots, n-2$, и предположим, что функции $\varkappa_{k}(x), k=0,1, \ldots, n-1$, продолжаются по непрерывности на $\overline{M_{k}}$. Последнее требование связано с тем, что возможны ситуации, когда $M_{k} \cap M_{k-1} \neq \emptyset$.

Замечание 2. Аналогично [9] в силу единственности волновой факторизации (см. [2]) можно убедиться, что функции $\varkappa_{k}(x), k=0,1, \ldots, m-1$, не зависят от выбора локальной системы координат.

Теорема 3. Предположим, что классический эллиптический символ $A(x, \xi)$ допускает $k$ волновую факторизацию относительно конусов $C^{m-k}$ с индексами $\varkappa_{k}(x), k=0,1, \ldots, m-2$, удовлетворяющими условию

$$
\left|\varkappa_{k}(x)-s\right|<\frac{1}{2} \quad \forall x \in M_{k}, \quad k=0,1, \ldots, m-1 .
$$

Тогда оператор $A: H^{s}(M) \rightarrow H^{s-\alpha}(M)$ фредгольмов.

Замечание 3. Если эллиптичность нарушается на подмногообразиях $M_{k}$, рассматриваются модификации оператора $A$ с привлечением граничных или кограничных операторов (см. $[2,3])$. В частности, это происходит, когда нарушается одно из условий (3).

\section{СПИСОК ЛИТЕРАТУРЫ}

1. Васильев В. Б. Регуляризация многомерных сингулярных интегральных уравнений в негладких областях// Тр. Моск. мат. о-ва. - 1998. - 59. - С. 73-105.

2. Васильев В. Б. Мультипликаторы интегралов Фурье, псевдодифференциальные уравнения, волновая факторизация, краевые задачи. - М.: УРСС, 2010.

3. Васильев В. Б. Модельные эллиптические краевые задачи для псевдодифференциальных уравнений в канонических негладких областях // Тр. семин. им. И. Г. Петровского. $-2016 .-31$. - С. 3-16.

4. Назаров С. А., Пламеневский Б. А. Эллиптические задачи в областях с кусочно гладкой границей. М.: Наука, 1991.

5. Пламеневский Б. А. Разрешимость алгебры псевдодифференциальных операторов с кусочно гладкими коэффициентами на гладком многообразии// Алгебра и анализ. - 2009. - 21, № 2. - С. 214-263.

6. Сили P. Т. Интегродифференциальные операторы на векторных расслоениях// Математика. - 1968. -11 , № 2. - C. 57-97. 
7. Симоненко И. Б. Локальный метод в теории инвариантных относительно сдвига операторов и их огибающих. - Ростов-на-Дону: ЦВВР, 2007.

8. Хъюзмоллер Д. Расслоенные пространства. - М.: Мир, 1970.

9. Эскин Г. И. Краевые задачи для эллиптических псевдодифференциальных уравнений. - М.: Наука, 1973.

10. Dynin A. S. Inversion problem for singular integral operators: $C^{*}$-approach// Proc. Natl. Acad. Sci. USA. - 1978. - 75. - P. 4668-4670.

11. Kottke C., Melrose R. B. Generalized blow-up of corners and fiber products// Trans. Am. Math. Soc. 2015. - 367, № 1. - P. 651-705.

12. Nazaikinskii V., Schulze B. W., Sternin B. The localization problem in index theory of elliptic operators. Pseudo-Differential Operators. Theory and Applications. — Basel: Birkhäuser, 2014.

13. Nistor $V$. Analysis on singular spaces: Lie manifolds and operator algebras// J. Geom. Phys. — 2016. 105. - P. 75-101.

14. Schulze B. W., Sternin B., Shatalov V. Differential Equations on Singular Manifolds: Semiclassical Theory and Operator Algebras. - Berlin: Wiley, 1998.

15. Vasilyev $V$. B. Asymptotical analysis of singularities for pseudo differential equations in canonical nonsmooth domains// in: Integral Methods in Science and Engineering. Computational and Analytic Aspects (Constanda C., Harris P. J., eds.). — Boston: Birkhäuser, 2011. — P. 379-390.

Васильев Владимир Борисович

Белгородский национальный исследовательский университет

E-mail: vbv57@inbox.ru 